\title{
Systematics of threshold incident energy for deep sub-barrier fusion hindrance
}

\author{
Takatoshi Ichikawa, ${ }^{1}$ Kouichi Hagino, ${ }^{2}$ and Akira Iwamoto ${ }^{3}$ \\ ${ }^{1}$ RIKEN, Wako, Saitama 351-0198, Japan \\ ${ }^{2}$ Department of Physics, Tohoku University, Sendai 980-8578, Japan \\ ${ }^{3}$ Japan Atomic Energy Agency, Tokai-mura, Naka-gun, Ibaraki 319-1195, Japan
}

(Dated: September 16, 2021)

\begin{abstract}
We systematically evaluate the potential energy at the touching configuration for heavy-ion reactions using various potential models. We point out that the energy at the touching point, especially that estimated with the Krappe-Nix-Sierk (KNS) potential, strongly correlates with the threshold incident energy for steep fall-off of fusion cross sections observed recently for several systems at extremely low energies. This clearly indicates that the steep fall-off phenomenon can be attributed to the dynamics after the target and projectile touch with each other, e.g., the tunneling process and the nuclear saturation property in the overlap region.
\end{abstract}

PACS numbers: 25.60.Pj, 24.10.Eq, 25.70Jj,25.70.-z

Recently, for medium-heavy mass systems, it has become possible to measure fusion cross sections down to extremely low incident energies. In those measurements, unexpected steep fall-off of fusion cross sections, as compared to a standard theoretical calculation, have been observed at deep subbarrier energies [1, 2, 3, 4, 5, 6]. Although the steep fall-off phenomenon, referred to as the fusion hindrance, may be accounted for if one uses an anomalously large diffuseness parameter in the Woods-Saxon potential [7], the physical origin of the phenomenon has yet to be clarified [8].

One important aspect of fusion reactions at deep subbarrier energies is that the inner turning point of the potential may be located far inside the touching point of the colliding nuclei. We show this schematically in Fig. 1. At energies close to the Coulomb barrier, the inner turning point is still far outside of the touching point [9] (see the line (i) in Fig. 1). At these energies, one usually assumes that a compound nucleus is automatically formed once the projectile penetrates the Coulomb barrier, due to the strong nuclear attractive force in the classically allowed region. In contrast, at energies below the potential energy at the touching point, $V_{\text {Touch }}$, the inner turning point appears more inside of the touching point (see the line (ii) in Fig. 11. That is, the projectile nucleus is still in the classically forbidden region when the two colliding nuclei touch with each other. After the touching, an elongated composite system is formed, which evolves in the classically forbidden region towards a compound nucleus by overlapping between the projectile-like and the target-like fragments. Since this involves the penetration of the residual Coulomb barrier, naturally the fusion cross sections are hindered by the tunneling factor.

In this paper, we evaluate the potential energy at the touching configuration for several systems, and investigate whether the dynamics after the touching point is responsible for the steep fall-off phenomenon. In this respect, it is interesting to notice that the authors of Refs. [1, 4] have argued that the steep fall-off phenomenon systematically takes place below a certain threshold incident energy, $E_{s}$. We will show below that there is a strong correlation between the touching energy $V_{\text {Touch }}$ and the threshold energy $E_{s}$, indicating that the density overlap in the classically forbidden region indeed plays an important role. We mention that one would have to settle

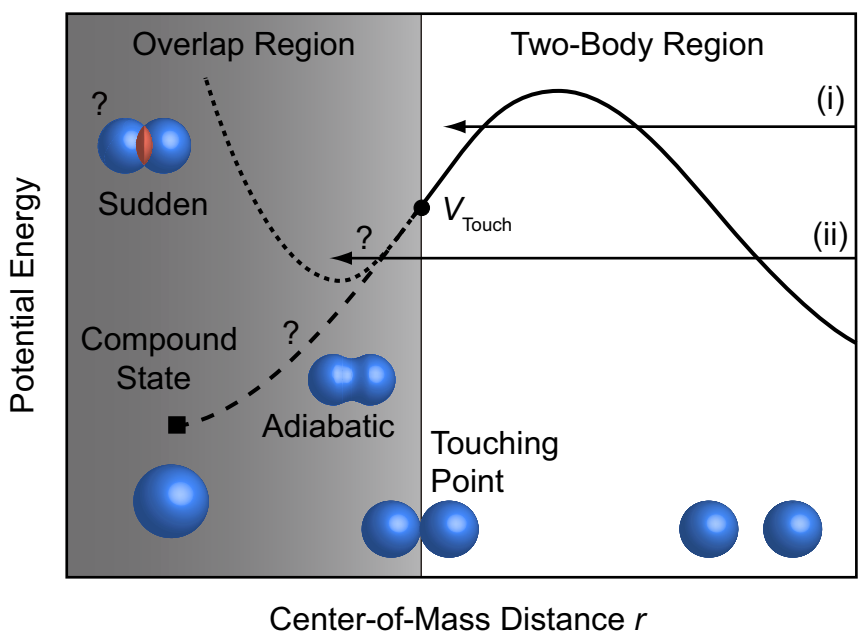

FIG. 1: (Color online) Schematic picture for heavy-ion sub-barrier fusion reactions. The filled circle denotes the energy at the touching point, $V_{\text {Touch }}$.

a model in the overlap region, such as the adiabatic or sudden models, or some combination of these two, in order to clarify the whole dynamics of deep subbarrier fusion reactions. However, our analysis is independent of these modellings, since both the adiabatic and the sudden approaches provide a similar potential energy to each other as long as the touching point is concerned.

In order to estimate the potential energy at the touching point, $r_{\text {touch }}=R_{P}+R_{T}$, we employ the Krappe-Nix-Sierk (KNS) [10], the Bass [11], the proximity [12] and the AkyüzWinther (AW) [13] models. Assuming the spherical shape for both the projectile and target nuclei, the KNS potential energy at the touching point reads

$$
V_{\mathrm{KNS}}^{(N)}=-D\left(4+\frac{r_{\mathrm{touch}}}{a}-\frac{f\left(R_{T} / a\right)}{g\left(R_{T} / a\right)}-\frac{f\left(R_{P} / a\right)}{g\left(R_{P} / a\right)}\right),
$$

where the functions $f$ and $g$ are defined as $f(x)=x^{2} \sinh (x)$ and $g(x)=x \cosh (x)-\sinh (x)$, respectively. In this model, the nuclear radius is given by $R=r_{0} A^{1 / 3}$, and the depth constant 
TABLE I: Potential energy at the touching configuration calculated by various theoretical models for the systems discussed in Ref. [5]. In the second column, $Z_{T} Z_{P}$ is the charge product of the system, while $\mu$ is the reduced mass. $X_{\text {eff }}$ is the effective fissility parameter defined as $X_{\text {eff }}=Z_{T} Z_{P} /\left[A_{T}^{1 / 3} A_{P}^{1 / 3}\left(A_{P}^{1 / 3}+A_{T}^{1 / 3}\right)\right] / 12.6$, while $E_{S}$ is the experimental energy at which the astrophysical S-factor has the maximum [5]. $V_{\mathrm{KNS}}, V_{\mathrm{Prox}}, V_{\mathrm{Bass}}$ and $V_{\mathrm{AW}}$ denote the results of the Krappe-Nix-Sierk, the proximity, the Bass and the Akyüz-Winther models, respectively.

\begin{tabular}{|c|c|c|c|c|c|c|c|}
\hline System & $\begin{array}{c}Z_{T} Z_{P} \sqrt{\mu} \\
\left(\mathrm{MeV}^{1 / 2} / c\right)\end{array}$ & $X_{\text {eff }}$ & $\begin{array}{c}E_{S} \\
(\mathrm{MeV})\end{array}$ & $\begin{array}{c}V_{\mathrm{KNS}} \\
(\mathrm{MeV})\end{array}$ & $\begin{array}{c}V_{\text {Prox }} \\
(\mathrm{MeV}) \\
\end{array}$ & $\begin{array}{c}V_{\text {Bass }} \\
(\mathrm{MeV})\end{array}$ & $\begin{array}{c}V_{\mathrm{AW}} \\
(\mathrm{MeV})\end{array}$ \\
\hline \multicolumn{8}{|l|}{ (Type I) } \\
\hline${ }^{90} \mathrm{Zr}+{ }^{90} \mathrm{Zr}$ & 10733 & 0.705 & $175 \pm 1.8$ & 179.9 & 169.6 & 167.6 & 175.2 \\
\hline${ }^{90} \mathrm{Zr}+{ }^{89} \mathrm{Y}$ & 10436 & 0.692 & $171 \pm 1.7$ & 175.2 & 164.8 & 162.4 & 170.4 \\
\hline${ }^{90} \mathrm{Zr}+{ }^{92} \mathrm{Zr}$ & 10792 & 0.698 & $171 \pm 1.7$ & 179.1 & 168.8 & 166.4 & 174.4 \\
\hline${ }^{58} \mathrm{Ni}+{ }^{58} \mathrm{Ni}$ & 4222 & 0.536 & $94 \pm 0.9$ & 93.4 & 80.8 & 79.2 & 87.5 \\
\hline${ }^{60} \mathrm{Ni}+{ }^{89} \mathrm{Y}$ & 6537 & 0.592 & $123 \pm 1.2$ & 125.4 & 113.6 & 111.1 & 119.8 \\
\hline${ }^{32} \mathrm{~S}+{ }^{89} \mathrm{Y}$ & 3026 & 0.457 & $72.6 \pm 0.7$ & 72.2 & 59.7 & 56.7 & 65.4 \\
\hline \multicolumn{8}{|l|}{ (Type II) } \\
\hline${ }^{64} \mathrm{Ni}+{ }^{100} \mathrm{Mo}$ & 7343 & 0.582 & $121 \pm 1.2$ & 131.7 & 120.0 & 115.9 & 126.2 \\
\hline${ }^{64} \mathrm{Ni}+{ }^{64} \mathrm{Ni}$ & 4435 & 0.486 & $87.3 \pm 0.9$ & 89.0 & 76.1 & 71.9 & 82.9 \\
\hline \multicolumn{8}{|l|}{ (Type III) } \\
\hline${ }^{48} \mathrm{Ca}+{ }^{48} \mathrm{Ca}$ & 1960 & 0.331 & $48.1 \pm 0.9$ & 42.2 & 27.7 & 21.9 & 35.4 \\
\hline${ }^{28} \mathrm{Si}+{ }^{64} \mathrm{Ni}$ & 1729 & 0.364 & $47.3 \pm 0.9$ & 43.9 & 30.5 & 27.1 & 36.7 \\
\hline${ }^{16} \mathrm{O}+{ }^{76} \mathrm{Ge}$ & 930.5 & 0.282 & $27.6 \pm 0.8$ & 26.1 & 13.1 & 9.6 & 18.3 \\
\hline \multicolumn{8}{|l|}{ (Type IV) } \\
\hline${ }^{16} \mathrm{O}+{ }^{16} \mathrm{O}$ & 181.0 & 0.159 & $7.1 \pm 0.8$ & 2.2 & -11.4 & -13.4 & -5.4 \\
\hline${ }^{12} \mathrm{O}+{ }^{16} \mathrm{O}$ & 125.7 & 0.137 & $<6.2$ & 0.2 & -13.2 & -14.8 & -7.4 \\
\hline${ }^{12} \mathrm{O}+{ }^{14} \mathrm{~N}$ & 106.8 & 0.129 & $<5.0$ & -0.5 & -13.9 & -15.4 & -8.1 \\
\hline${ }^{12} \mathrm{O}+{ }^{13} \mathrm{C}$ & 89.9 & 0.114 & $<4.0$ & -1.5 & -14.9 & -16.6 & -9.3 \\
\hline${ }^{11} \mathrm{O}+{ }^{12} \mathrm{C}$ & 71.9 & 0.104 & $<3.0$ & -2.2 & -15.5 & -16.9 & -9.9 \\
\hline${ }^{10} \mathrm{~B}+{ }^{10} \mathrm{~B}$ & 55.9 & 0.099 & $<1.9$ & -2.2 & -15.3 & -16.0 & -9.9 \\
\hline
\end{tabular}

$D$ by

$$
D=\frac{4 \sqrt{c_{s}^{(T)} c_{s}^{(P)}} a^{3}}{r_{0}^{2} r_{\text {touch }}} g\left(R_{T} / a\right) g\left(R_{P} / a\right) e^{-r_{\text {touch }} / a},
$$

where the effective surface energy constant $c_{s}$ is given by $c_{s}=a_{s}\left(1-\kappa_{s} I^{2}\right)$ with $I=(N-Z) / A$. We take the parameters to be $a=0.68 \mathrm{fm}, a_{s}=21.33 \mathrm{MeV}$ and $\kappa_{s}=2.378$ from FRLDM2002 [12], except for the radius parameter for which we slightly adjust to be $r_{0}=1.2 \mathrm{fm}$ in order to fit the experimental fusion cross sections for the ${ }^{64} \mathrm{Ni}+{ }^{64} \mathrm{Ni}$ reaction at energies above the Coulomb barrier.

The proximity potential energy at the touching point is given by

$$
V_{\mathrm{Prox}}^{(N)}=-1.7818 \frac{b \bar{R} a_{2}}{r_{0}^{2}}-3.00,
$$

where $\bar{R}=R_{T} R_{P} /\left(R_{T}+R_{P}\right)$. In this model, the nuclear radius is given by

$$
R=R_{00}\left(1-\frac{7}{2} \frac{b^{2}}{R_{00}^{2}}-\frac{49}{8} \frac{b^{4}}{R_{00}^{4}}\right)+\frac{N}{A} t
$$

with

$$
\begin{aligned}
R_{00} & \left.=1.240 A^{1 / 3}[1+1.646 / A-0.191(A-2 Z) / A)\right] \\
t & =\frac{3}{2} r_{0} \cdot \frac{J(N-Z) / A-\frac{1}{12} c_{1} Z A^{-1 / 3}}{Q+\frac{9}{4} J A^{-1 / 3}}
\end{aligned}
$$

The value of the parameters are taken to be $b=1 \mathrm{fm}, r_{0}=1.14$ fm, $J=32.65 \mathrm{MeV}, c_{1}=0.757895 \mathrm{MeV}$ and $Q=35.4 \mathrm{MeV}$. The surface energy coefficient $a_{2}$ in Eq. (3) is given by $a_{2}=$ $18.36-Q\left(t_{T}^{2}+t_{P}^{2}\right) / 2 r_{0}^{2}$. In order to fit the experimental data, we use the same prescription as in Refs. [15, 16] and subtract $3.00 \mathrm{MeV}$ from the original proximity model (the last term in Eq. (3)).

The Bass potential energy at the touching point is given by

$$
V_{\text {Bass }}^{(N)}=-\bar{R}[\alpha+\beta]^{-1},
$$

where the parameters $\alpha$ and $\beta$ are taken as $\alpha=0.0300 \mathrm{MeV}^{-1}$ $\mathrm{fm}$ and $\beta=0.0061 \mathrm{MeV}^{-1} \mathrm{fm}$, respectively. In the Bass model, the nuclear radius is given by $R=1.16 A^{1 / 3}-1.39 A^{-1 / 3}$. The AW potential energy at the touching point, on the other hand, reads

$$
V_{\mathrm{AW}}^{(N)}=-8 \pi \gamma \bar{R} a,
$$

where the average surface tension $\gamma$ is given by $\gamma=$ $0.95\left[1-1.8\left(\frac{N_{T}-Z_{T}}{A_{T}}\right)\left(\frac{N_{P}-Z_{P}}{A_{P}}\right)\right] \mathrm{MeV} \mathrm{fm}^{-2}$. In this model, the nuclear radius is given by $R=\left(1.20 A^{1 / 3}-0.09\right) \mathrm{fm}$ and the diffuseness parameter $a$ is given by $a=0.855 \cdot[1+$ $\left.0.53\left(A_{T}^{-1 / 3}+A_{P}^{-1 / 3}\right)\right]^{-1} \mathrm{fm}$.

In order to estimate the total potential energy at the touching point, $V_{\text {Touch }}$, one has to add the Coulomb potential to the nuclear potential energies given by Eqs. (3) - (8). To this end, we use the Coulomb potential for two point charges, 


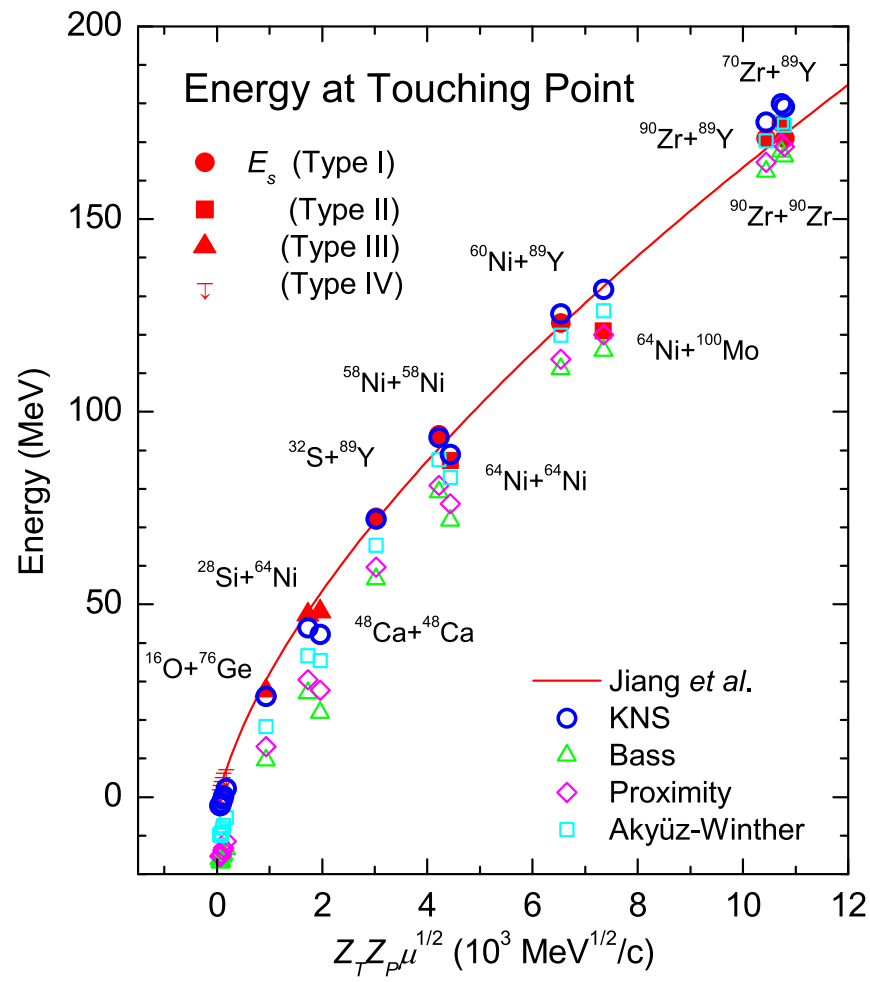

FIG. 2: (Color online) Potential energy at the touching point calculated by various theoretical models. The open circles, triangles, diamonds and squares denote the results of the Krappe-Nix-Sierk, the Bass, the Proximity and the Akyüz-Winther models, respectively. The solid line denotes the systematics proposed by Jiang et al. [5]. The filled circles, squares, triangles, and the horizontal lines show the experimental energy taken from Ref. [5] at which the astrophysical $\mathrm{S}$-factor has the maximum value.

$V^{(C)}=e^{2} Z_{T} Z_{P} / r_{\text {touch }}$, where the touching radius $r_{\text {touch }}$ is specified for each model for the nuclear potential, $V^{(N)}$. The resultant touching energy for the systems discussed in Ref. [5] is shown in Fig. 1 1 as a function of $Z_{T} Z_{P} \mu^{1 / 2}$, where $\mu$ is the reduced mass of the colliding nuclei. The results of the KNS, the Bass, the proximity and the AW models are denoted by the open circle, the open triangle, the open diamond and the open square, respectively. All the results are summarized in Table 1. These touching energies are compared with the energy $E_{S}$, at which the experimental fusion cross section is maximum when it is plotted in terms of the astrophysical S-factor [5]. These "experimental" energies $E_{s}$ are shown in Fig. 2 by the filled circles, the filled squares, the filled triangles, and the horizontal lines, depending on the types of the system as defined in Ref. [5]. Notice that the energy $E_{S}$ for the type III was estimated by extrapolation, and that for the type IV is only an upper limit. The systematics for the energy $E_{s}$ proposed by Jiang et al. [5] is also shown by the solid line.

Althogh the physical significance for the energy $E_{s}$ is not clear, because the $\mathrm{S}$-factor representation for fusion cross sections would be useful only at much lower energies than the lowest energies of the current measurements, at which the outer turning point is much larger than the inner turning point
TABLE II: The potential energy at the touching configuration calculated with the KNS model for the systems discussed in Refs. [2, 3, 4]. All of these systems are categolized as Type III.

\begin{tabular}{cccccc}
\hline \hline System & $\begin{array}{c}Z_{T} Z_{P} \sqrt{\mu} \\
\left(\mathrm{MeV}^{1 / 2} / c\right)\end{array}$ & $X_{\text {eff }}$ & $\begin{array}{c}E_{S} \\
(\mathrm{MeV})\end{array}$ & $\begin{array}{c}V_{\mathrm{KNS}} \\
(\mathrm{MeV})\end{array}$ & Ref. \\
\hline${ }^{34} \mathrm{~S}+{ }^{89} \mathrm{Y}$ & 3095 & 0.444 & 72.6 & 70.9 & {$[2]$} \\
${ }^{28} \mathrm{Si}+{ }^{58} \mathrm{Ni}$ & 1704 & 0.383 & 49 & 45.3 & {$[2]$} \\
${ }^{28} \mathrm{Si}+{ }^{62} \mathrm{Ni}$ & 1722 & 0.370 & 48.6 & 44.3 & {$[2]$} \\
${ }^{16} \mathrm{O}+{ }^{208} \mathrm{~Pb}$ & 2529 & 0.413 & 69.6 & 70.5 & {$[3]$} \\
${ }^{16} \mathrm{O}+{ }^{144} \mathrm{Sm}$ & 1882 & 0.384 & 57.7 & 54.6 & {$[3]$} \\
${ }^{19} \mathrm{~F}+{ }^{208} \mathrm{~Pb}$ & 3079 & 0.431 & 75.5 & 78.8 & {$[3]$} \\
${ }^{40} \mathrm{Ca}+{ }^{90} \mathrm{Zr}$ & 4210 & 0.524 & 93.2 & 93.6 & {$[3]$} \\
${ }^{50} \mathrm{Ti}+{ }^{208} \mathrm{~Pb}$ & 11454 & 0.683 & 181.2 & 191.9 & {$[3]$} \\
${ }^{58} \mathrm{Ni}+{ }^{60} \mathrm{Ni}$ & 4258 & 0.527 & $92 \pm 2$ & 92.5 & {$[4]$} \\
${ }^{58} \mathrm{Ni}+{ }^{64} \mathrm{Ni}$ & 4325 & 0.511 & $89 \pm 2$ & 91.1 & {$[4]$} \\
${ }^{58} \mathrm{Ni}+{ }^{74} \mathrm{Ge}$ & 5109 & 0.542 & $98.5 \pm 2.0$ & 103.9 & {$[4]$} \\
${ }^{64} \mathrm{Ni}+{ }^{74} \mathrm{Ge}$ & 5249 & 0.517 & $97.5 \pm 2.0$ & 101.5 & {$[4]$} \\
\hline \hline
\end{tabular}

(see e.g., Refs. [17, 18] for a discussion on the modified Sfactor, that takes into account the effect of the inner turning point), it is remarkable that the result of the KNS model follows closely to the energy $E_{S}$, and thus the systematics shown by the solid line (an exceptional case of ${ }^{64} \mathrm{Ni}+{ }^{100} \mathrm{Mo}$ will be discussed in the next paragraph). The good correspondence between $V_{\mathrm{KNS}}$ and $E_{s}$ may be due to the fact that the KNS model partly takes into account the saturation of nuclear matter when two nuclei come inside the Coulomb barrier[19] (in fact, the KNS model has been shown to be consistent with the energy density formalism with the Skyrme SkM* interaction [20,21]). The result of the AW potential is similar to that of the KNS model, although the deviation from $E_{s}$ is slightly larger. For the Bass and the proximity models, although the dependence of the touching energy $V_{\text {Touch }}$ on the parameter $Z_{T} Z_{P} \mu^{1 / 2}$ is similar to that of the KNS and the AW models, there is a large discrepancy between the touching energy $V_{\text {touch }}$ and the threshold energy $E_{s}$.

For the asymmetric ${ }^{64} \mathrm{Ni}+{ }^{100} \mathrm{Mo}$ reaction, the experimental threshold energy $E_{s}$ deviates largely from the systematics curve. The calculations with the KNS and AW models are consistent with the systematics curve but not to the value of $E_{s}$. In order to check how the touching energy $V_{\text {Touch }}$ compares with the threshold energy $E_{s}$ for other asymmetric systems, we also examine the ${ }^{16} \mathrm{O}+{ }^{208} \mathrm{~Pb}$ reaction. For this system, we find that the KNS model leads to the touching energy $V_{\text {Touch }}$ that is consistent with the experimental threshold energy $E_{s}$ [3] (see Table II). Therefore, it is unlikely that the large difference between $V_{\text {Touch }}$ and $E_{s}$ for the ${ }^{64} \mathrm{Ni}+{ }^{100} \mathrm{Mo}$ system can be attributed to the model assumption of the KNS potential. Notice that for this system, there may exist some peculiar nuclear structure effect, because the coupled-channels calculation reported in Ref. [22] does not seem to account well for the experimental fusion cross sections even above the threshold energy $E_{s}$. A further investigation is necessary for this system concerning the threshold energy.

In order to see more clearly the correlation between $E_{s}$ and $V_{\mathrm{KNS}}$, the lower panel of Fig. 3 shows these energies as 


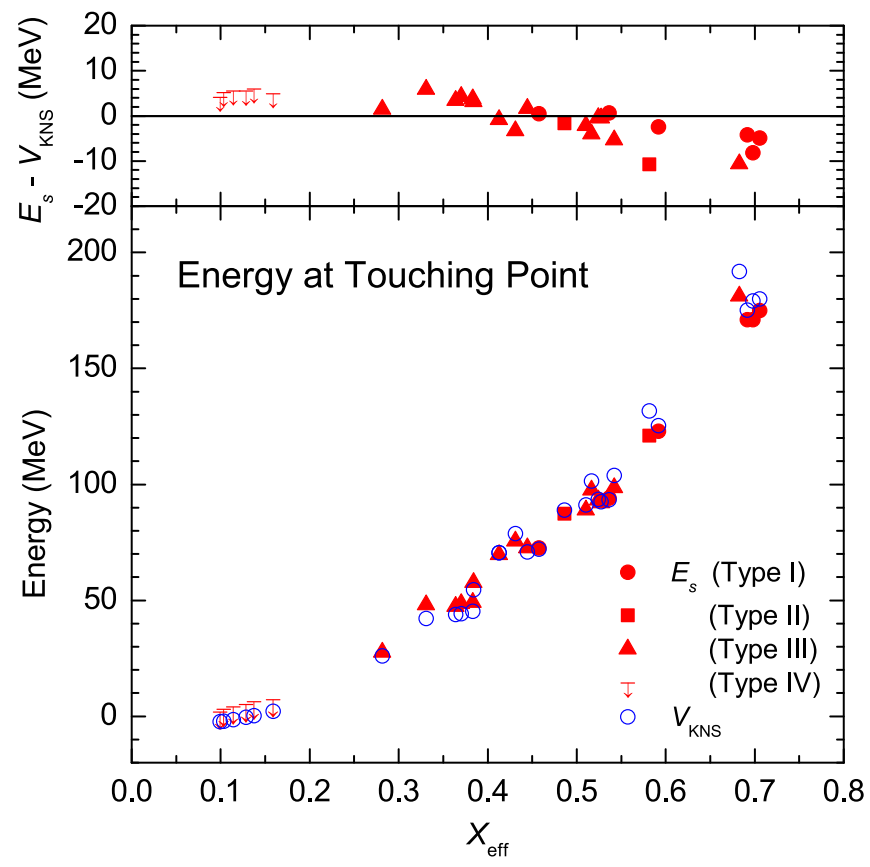

FIG. 3: (Color online) Same as Fig. 2, but as a function of the effective fissility parameter defined in Ref. [11]. The upper panel shows the difference between $E_{S}$ and $V_{\mathrm{KNS}}$.

a function of the effective fissility parameter $X_{\text {eff }}$ defined as $X_{\text {eff }}=Z_{T} Z_{P} /\left[A_{T}^{1 / 3} A_{P}^{1 / 3}\left(A_{P}^{1 / 3}+A_{T}^{1 / 3}\right)\right] / 12.6[11]$. The figure includes also a few more systems than shown in Fig. 2, which are taken from Refs. [2, 3, 4] (see Table II for the additional data). With this representation, all the data points distribute more uniformly than in Fig. 22 The difference between $E_{s}$ and $V_{\mathrm{KNS}}$ is also shown in the upper panel of Fig. 3. One observes that the difference between $E_{s}$ and $V_{\mathrm{KNS}}$ is indeed small except for large $X_{\text {eff }}$, clearly indicating that there is a strong correlation between these two values. The large discrepancy for systems with large $X_{\text {eff }}$ may be due to the ambiguity of the experimental data, because the measurements were only for the fusion-evaporation cross sections and the fusion-fission cross sections were estimated using the statistical model [2, 3, 4] ].

In summary, we have shown that the potential energy at the touching point strongly correlates with the threshold incident energies for the steep fall-off of the fusion cross sections. The systematics of the threshold energy can be rather naturally explained by the present approach in terms of the touching energy. This strongly suggests that the overlap process after the touching is responsible for the steep fall-off of the fusion cross section. For such overlap process, the sudden and adiabatic approaches have been often employed [23, 24]. In the former, the frozen density approximation while overlapping with the colliding nuclei is applied, and in the latter, the dynamical change in the density of the colliding nuclei is taken into account. These two approaches are in the opposite limit to each other, and there is not yet a definite consensus regarding which limit better describes the realistic situation at deep subbarrier energies. In this respect, the threshold energy discussed in this paper will provide a useful constraint to modelling of the overlap process as the touching configuration is a doorway of such process.

\section{Acknowledgments}

This work was supported by the Grant-in-Aid for Scientific Research, Contract No. 19740115 from the Japanese Ministry of Education, Culture, Sports, Science, and Technology.
[1] C. L. Jiang, H. Esbensen, K. E. Rehm, B. B. Back, R. V. F. Janssens, J. A. Caggiano, P. Collon, J. Greene, A. M. Heinz, D. J. Henderson, et al., Phys. Rev. Lett. 89, 052701 (2002).

[2] C. L. Jiang, K. E. Rehm, R. V. F. Janssens, H. Esbensen, I. Ahmad, B. B. Back, P. Collon, C. N. Davids, J. P. Greene, D. J. Henderson, et al., Phys. Rev. Lett. 93, 012701 (2004).

[3] C. L. Jiang, H. Esbensen, B. B. Back, R. V. F. Janssens, and K. E. Rehm, Phys. Rev. C 69, 014604 (2004).

[4] C. L. Jiang, K. E. Rehm, H. Esbensen, R. V. F. Janssens, B. B. Back, C. N. Davids, J. P. Greene, D. J. Henderson, C. J. Lister, R. C. Pardo, et al., Phys. Rev. C 71, 044613 (2005).

[5] C. L. Jiang, B. B. Back, H. Esbensen, R. V. F. Janssens, and K. E. Rehm, Phys. Rev. C 73, 014613 (2006).

[6] C. L. Jiang, B. B. Back, H. Esbensen, R. V. F. Janssens, Ş. Mişicu, K. E. Rehm, P. Collon, C. N. Davids, J. Greene, D. J. Henderson, et al., Physics Letters B 640, 18 (2006).

[7] K. Hagino, N. Rowley, and M. Dasgupta, Phys. Rev. C 67, 054603 (2003).

[8] M. Dasgupta, D. J. Hinde, C. Low, and J. O. Newton, AIP Conf. Proc. 853, 21 (2006).

[9] M. Dasgupta, D. J. Hinde, N. Rowley, and A. M. Stefanini, Annu. Rev. Nucl. Part. Sci. 48, 401 (1998).
[10] H. J. Krappe, J. R. Nix, and A. J. Sierk, Phys. Rev. C 20, 992 (1979).

[11] R. Bass, Nuclear reactions with heavy ions (Springer-Verlag New York, 1980).

[12] W. D. Myers and W. J. Świạtecki, Phys. Rev. C 62, 044610 (2000).

[13] R. A. Broglia and A. Winther, Heavy Ion Reactions, Lecture Notes, Volume I: The Elementary Processes (Addison-Wesley, Reading, MA, 1991).

[14] P. Möller, A. J. Sierk, and A. Iwamoto, Phys. Rev. Lett. 92, 072501 (2004).

[15] K. Siwek-Wilczyńska and J. Wilczyński, Phys. Rev. C 69, 24611 (2004).

[16] W. J. Świa̧tecki, K. Siwek-Wilczyńska, and J. Wilczyński, Phys. Rev. C 71, 014602 (2005).

[17] M.G. Mazarakis and W.E. Stephens, Phys. Rev. C7, 1280 (1973).

[18] T. Spillane et al., Phys. Rev. Lett. 98, 122501 (2007).

[19] T. Ichikawa, K. Hagino, and A. Iwamoto, to be published.

[20] L. C. Vaz, J. M. Alexander, and G. R. Satchler, Phys. Rep. 69, 373 (1981).

[21] V. Yu. Denisov and W. Nörenberg, Eur. Phys. J. A 15, 375 
(2002).

[22] H. Esbensen, Phys. Rev. C 72, 054607 (2005).

[23] A. B. Balantekin and N. Takigawa, Rev. Mod. Phys. 70, 77
(1998).

[24] K. Hagino and K. Washiyama, AIP Conf. Proc. 853, 86 (2006). 